\title{
Physiologically Based Pharmacokinetic Modeling: Methodology, Applications, and Limitations with a Focus on Its Role in Pediatric Drug Development
}

\author{
Feras Khalil and Stephanie Läer \\ Department of Clinical Pharmacy and Pharmacotherapy, Heinrich-Heine University of Düsseldorf, 40225 Düsseldorf, Germany \\ Correspondence should be addressed to Stephanie Läer, stephanie.laeer@uni-duesseldorf.de
}

Received 15 October 2010; Revised 4 January 2011; Accepted 3 March 2011

Academic Editor: Ronald E. Baynes

Copyright (c) 2011 F. Khalil and S. Läer. This is an open access article distributed under the Creative Commons Attribution License, which permits unrestricted use, distribution, and reproduction in any medium, provided the original work is properly cited.

\begin{abstract}
The concept of physiologically based pharmacokinetic (PBPK) modeling was introduced years ago, but it has not been practiced significantly. However, interest in and implementation of this modeling technique have grown, as evidenced by the increased number of publications in this field. This paper demonstrates briefly the methodology, applications, and limitations of PBPK modeling with special attention given to discuss the use of PBPK models in pediatric drug development and some examples described in detail. Although PBPK models do have some limitations, the potential benefit from PBPK modeling technique is huge. PBPK models can be applied to investigate drug pharmacokinetics under different physiological and pathological conditions or in different age groups, to support decision-making during drug discovery, to provide, perhaps most important, data that can save time and resources, especially in early drug development phases and in pediatric clinical trials, and potentially to help clinical trials become more "confirmatory" rather than "exploratory".
\end{abstract}

\section{Introduction}

Although the concept of physiologically based pharmacokinetic (PBPK) modeling was introduced years ago, it has not achieved the scope of development and implementation that it deserves. Still, interest in and use of this modeling technique have grown over the last few years, as evidenced by the large increase in publications in this field [1]. The initiation of the Pediatric Exclusivity Program in the USA and Europe may also contribute to wider use of this modeling technique, as PBPK models can bridge pediatric and adult pharmacology. The general concept of PBPK modeling is to mathematically describe relevant physiological, physicochemical, and biochemical processes that determine the pharmacokinetic behavior of a compound in as much detail as is appropriate or needed. In order to accomplish this, principles of physiology and anatomy is employed to represent the species to be modeled as a structure composed of physiologically relevant compartments, where each compartment often represents a single organ or tissue. Following the anatomical structure of the organism, these compartments are interconnected via the blood circulation loop, and the mass-balance equations for each compartment describing the fate of the substance within it are established. To solve these equations, the PBPK model uses physiological and substance-specific parameters. In the end, PBPK models can describe and/or predict drug pharmacokinetics in certain individuals, or under certain physiological or pathological conditions, where the primary result of a simulation is a set of concentration-time curves illustrating the temporal behavior of the drug in blood/plasma and/or other relevant organs. Figure 1 illustrates the concept of building a PBPK model.

A number of articles reviewing PBPK modeling techniques have been published; however, they cover the subject from a different perspective than this paper and/or possess a different aim and scope. Chen and Gross [2] and Himmelstein and Lutz [3] discuss the rationale and history behind the development of PBPK models and report examples of the first PBPK models. Other reviews focus mainly on the structure and methodology of PBPK modeling $[1,4,5]$ or on its implementation in certain areas-for example, to guide research and development of new drugs [6], to predict 
drug pharmacokinetics in pediatric populations $[7,8]$, or to predict drug interactions [9]. Even in articles that resemble our way of approaching the subject, the limitations of PBPK modeling are not clearly discussed, and either the scope of the presented applications is different [10], or less emphasis is placed on demonstrating the potential use of PBPK models to save time and effort in pediatric drug clinical investigations $[11,12]$. Therefore, our objective is to provide researchers and scientists who are interested in using the PBPK modeling technique with a simplified and general overview of the structure of PBPK models as well as the various software packages that can be used in model development. We have also highlighted some of the technique's weaknesses and provided an overview of its various applications with an emphasis on recent examples that show the potential of PBPK models, especially in pediatric drug development where PBPK modeling arises as a valuable method that can aid in conducting research in children.

\section{Discussion}

2.1. PBPK Model Development. Generally, the five major steps in PBPK model development include (1) specifying general model structure, (2) specifying tissue model, (3) writing model equations, (4) defining model parameterization, and (5) simulations and/or parameter estimation.

2.1.1. General Model Structure Specification. PBPK models emulate the structure of the living organism being studied and represent the various organs and tissues as compartments in the model that are connected via a blood circulation loop which is subdivided into arterial and venous pools. The natural basis for the choice of compartments is the available data concerning the anatomy and physiology of the biological system from the cellular level to the whole body. However, this does not itself determine how many body regions, or compartments, are needed, since the important aspects of the drug's pharmacokinetic events must be evaluated. The choice also depends upon the model's purpose and the physicochemical (binding, lipid solubility, and ionization) and pharmacological (mechanism of transport, site[s] of action) properties of the modeled drug [14]. For example, if the drug is not lipid soluble, the details of the adipose tissues of the body are not particularly important; or if only the absorption of the drug is of interest, then a model that includes only those body tissues or organs involved in the absorption process may be sufficient. The complexity of the models and the amount of incorporated information increase with the increasing number of represented tissues/organs; however, due to the fact that the main features of drug distribution can often be described with models that have surprisingly few details, a common strategy in structuring PBPK models, called "lumping," is implemented $[15,16]$. Tissues that share similar physiological, physicochemical, and biochemical properties are grouped as one compartment, while tissues with distinct properties-such as the liver, where metabolism occurs, or target tissues-are separated from the lumped compartments. Eventually, PBPK models do vary, ranging from partial-body PBPK models which include certain body systems or tissues, to whole-body PBPK models where almost all body tissues are included and represented either as separated or lumped compartments.

2.1.2. Tissues/Organs Model Specification. The model of each particular tissue or organ must subsequently be specified (i.e., subcompartments representing each organ/tissue must be determined). The vast majority of PBPK models involve one to four compartments for each tissue or organ. The compartmentalization decision is based on existing information regarding tissue kinetics and the biochemical process involved once the drug gets into the tissue [1]. It is important to distinguish between the different assumptions made at this level. A perfusion rate-limited tissue model is assigned under the assumption that on entry with the blood circulation, the drug distributes freely and instantly across the membranes without diffusion barriers; thus, it is the rate of delivery by the blood that is rate limiting. If diffusion barriers to the distribution of a compound in the tissue are presumed and can be physiologically identified-for example, capillary membranes (such as the blood-brain barrier for some hydrophilic molecules) or cellular membranes, or both-the more complex permeability rate-limited tissue model will be assigned with at least a two-compartment tissue structure. Furthermore, the assumption of a well-stirred model is that there is no concentration gradient within a tissue/organ compartment. This contrasts with the dispersion model, in which a diffusion barrier cannot be identified but concentration gradients nevertheless exist. Examples of a perfusion ratelimited, well-stirred, one-compartment tissue model and a permeability rate-limited, two-compartment tissue model are given in Figure 2.

2.1.3. Writing the PBPK Model Equations. The PBPK model equations are derived from the law of mass action-that is, they are mass balance equations, as the kinetic processes are mass transfer phenomena. Four types of mathematical descriptions of the tissues within PBPK models have been used $[4,15]$ : (i) algebraic descriptions, which are used when the processes are assumed to equilibrate instantly and can be considered static (e.g., alveolar and inhaled air concentrations), (ii) linear ordinary differential, which are the most commonly used descriptions in describing dynamic pharmacokinetic processes, (iii) nonlinear differential, which are used to represent non-linear processes within a particular tissue (e.g., concentration-dependent clearance and/or binding), and (iv) partial differential, which are used with the dispersion tissue model. Figure 2 shows examples of different differential equations that can be used in a PBPK model.

2.1.4. PBPK-Model Parameterization. Once model equations are written, their parameters must be specified and/or estimated. The parameters for incorporation into PBPK models are generally either physiological or compound dependent.

Physiological parameters characterize the anatomical structure and physiological processes of the species being 


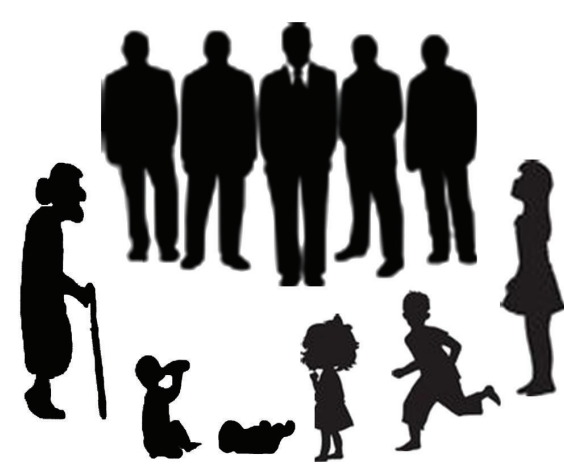

(a)

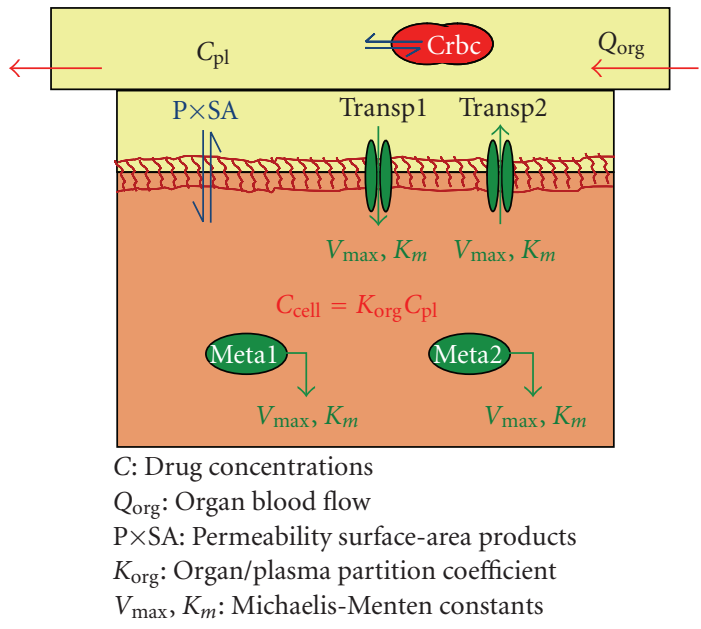

(c)

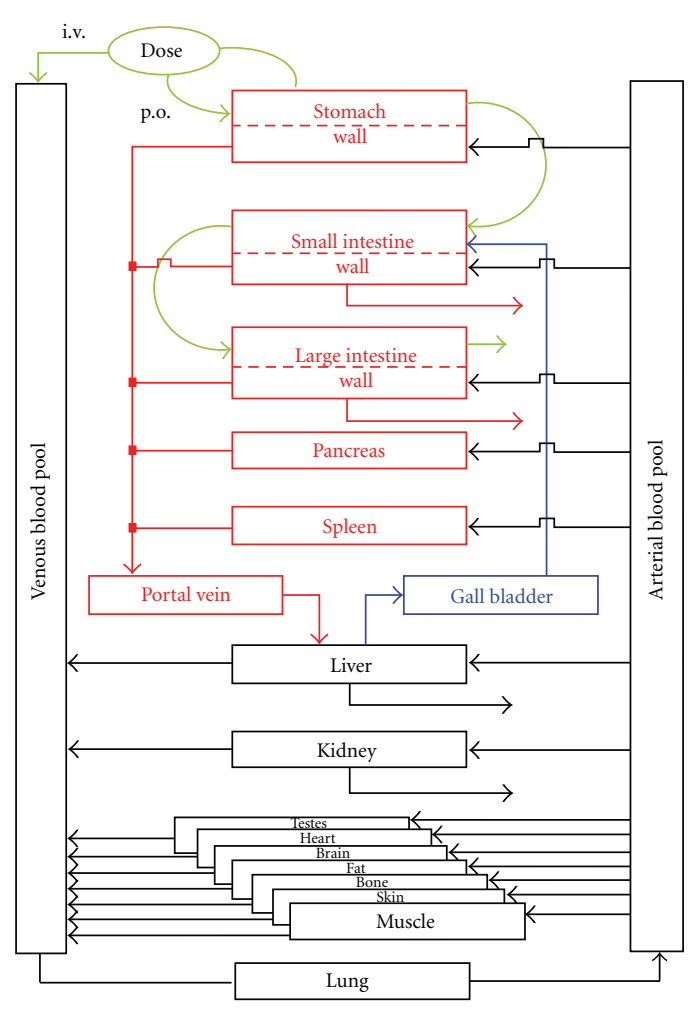

(b)
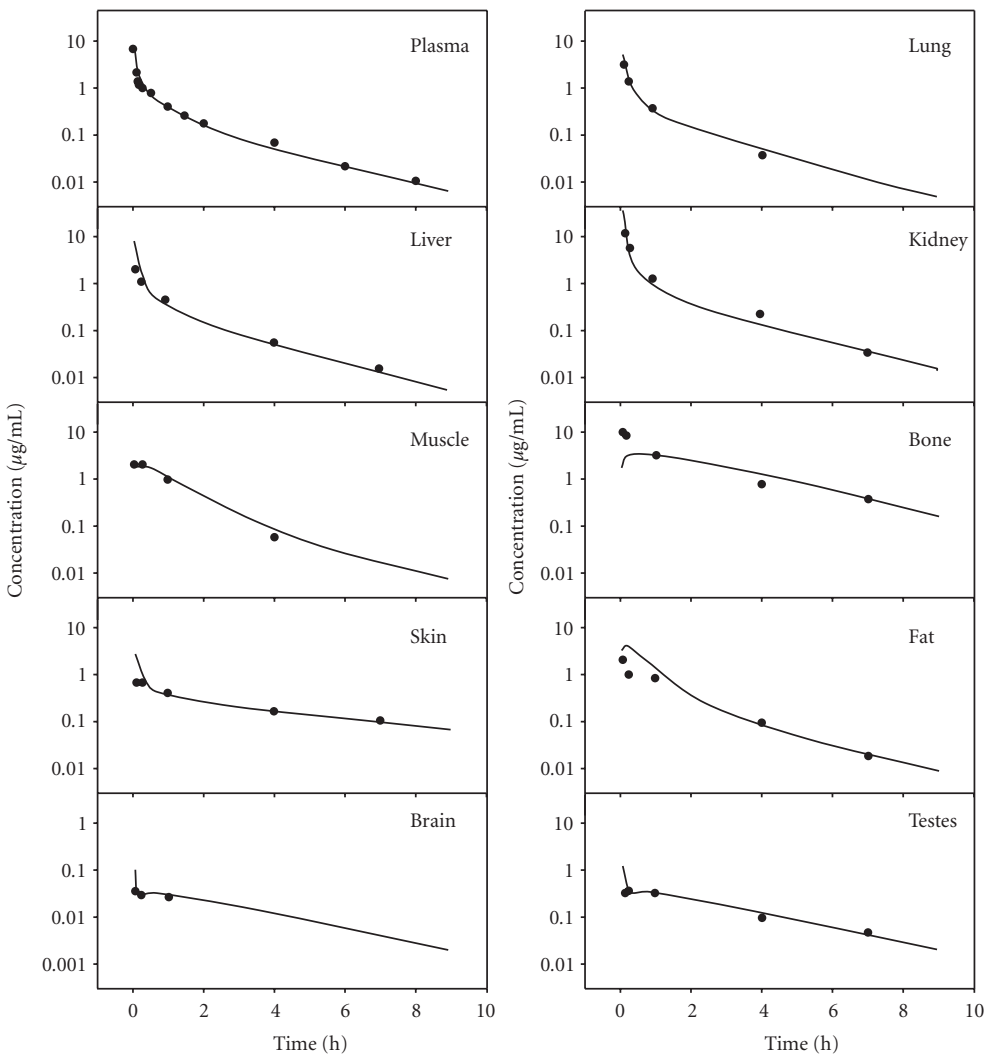

(d)

FIgURE 1: The concept for building a PBPK model modified according to Willmann et al. [13]. (a) Organisms (e.g., humans of different ages or populations) are the basis for the model. (b) The organism is divided into a number of compartments, each representing a single organ. To describe the distribution of compounds in the body, the organs are connected via their arteries and veins to the arterial and venous blood pool. Intercompartmental mass transport occurs via organ-specific blood flow rates. The organs are mathematically connected. (c) Division of each organ into three subcompartments representing the vascular space with blood cells and the interstitial and cellular space. The interstitial space is assumed to be in direct contact with the plasma. The exchange of substances between the cellular and interstitial compartment can occur by permeation across the membranes via passive diffusion as well as active influx and efflux transport processes by saturable Michaelis-Menten (MM) kinetics with $V_{\max }$ and $K_{\mathrm{m}}$ as parameters. Metabolism of substances (Meta1 and Meta2) occurs via active enzymes (MM kinetics). Finally, the model consists of a large number of coupled differential equations. (d) Output of the model: concentration time curves for the substances shown are simulated and observed ciprofloxacin concentrations in various organs after ciprofloxacin $5 \mathrm{mg} / \mathrm{kg}$ was intravenously applied to a rat. 


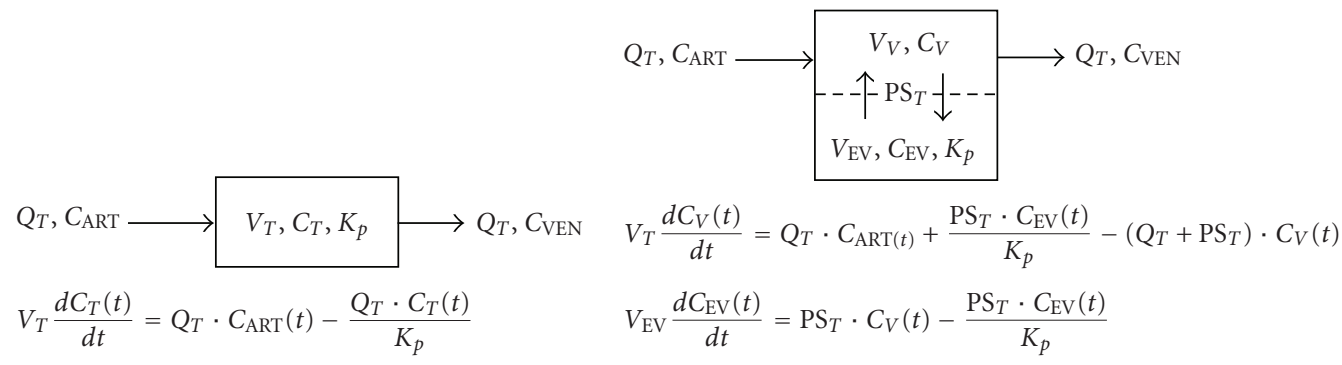

(a)

Figure 2: Diagrams and equations for a perfusion rate-limited, one-compartment model (a) and a permeability rate-limited, twocompartment model with the permeability at the vascular membrane (b) of noneliminating organs, adapted from Nestorov et al. [15]. $Q=$ blood flow; $C=$ concentration; $V=$ volume; $K p=$ tissue : plasma distribution coefficient; PS = permeability surface area coefficient; subscripts $T$, ART, VEN, $V$, and EV indicate tissue, arterial, venous, vascular compartment, and extravascular compartment, respectively.

modeled, parameters such as organ/tissue volumes, cardiac output and blood flows, tissue composition, surface area, $\mathrm{pH}$ values, and/or transit times for the gastrointestinal tract. The values of these parameters are known to vary among species and subjects or with age and physiologi$\mathrm{cal} /$ pathological state. Despite the large volume of available literature reporting such physiological data in numerous species_-notably, annals of the International Committee on Radiological Protection (ICRP) for human values [17] and Brown et al. for animal values [18] -allometric scaling (i.e., extrapolation from another species or another age group) is still used when various physiological parameters that are needed for a PBPK model are incomplete or entirely lacking. Although physiological parameters are most often assumed to be compound independent, drugs can sometimes affect the physiology of the biological system as seen, for example, in the change of cardiac output and blood flow due to the induction of anesthesia $[19,20]$. Therefore, the potential pharmacological effects of some compounds on the physiological variables must be accounted for in such circumstances.

The second set of parameters necessary for a PBPK model are compound dependent and include information such as permeability - surface area products $(\mathrm{P} \times \mathrm{SA})$ and partitioning of the substance between body tissues and the blood/plasma $\left(\mathrm{Kp}_{T}\right)$. These parameters can be obtained either from in vitro experiments, by extrapolating the experimental in vivo values from animals to humans, or by estimation/prediction using specific algorithms. A great deal of specialized PBPK modeling software includes such algorithms_for instance, those developed by Poulin and Theil, Rodgers and Rowland, or Schmitt [21-23] to calculate tissue:plasma partition coefficients. In the latter case, easily obtained compound characteristics such as molecular weight (MW), lipophilicity ( $\log P$ or $\log D$ value), ionization $\left(p K_{a}\right.$ value), and plasma fraction unbound $\left(f_{u}\right)$ are alternative inputs, as these algorithms use the previous information along with tissue composition (lipids, proteins, and water) to estimate partition coefficients.

Clearance is a compound/species-specific parameter that greatly influences the pharmacokinetic behavior of a drug; thus, a measure of clearance is a necessary PBPK model input. It is up to the researcher to decide how to provide the PBPK model with this information. An experimental blood or plasma clearance is often used as a direct input; however, as many PBPK models are able to perform in vitro-in vivo extrapolations (IVIVE), in vitro data can also be used-for example, data from in vitro experiments on microsomes or hepatocytes (in the form of half-lives or residual fractions). In the latter case, depending on the type of input parameter, PBPK models use additional information such as liver volume, liver blood flow, or microsomal binding to calculate the in vivo hepatic intrinsic clearance.

\subsubsection{PBPK Modeling Software for Simulation and Parameter} Estimation. In the last step, the entire system is coupled and equations that describe the model are coded in a particular software language for subsequent parameter estimation and/or simulation. Several commercial software tools for developing PBPK models are available; however, it is important to distinguish between general mathematical and engineering modeling software and specialized PBPK modeling software packages. General modeling software packages, such as MATLAB: [http://www.mathworks.com/ products/matlab/], ModelMaker: [http://www.modelkinetix .com/modelmaker/], Berkeley Madonna: [http://www.berkeleymadonna.com/], and acslX, [http://www.acslX.com], provide a programming language for the model code, numerical solutions for the ordinary differential equations that define the system being modeled, and a graphical output of the simulation results. These software packages offer much flexibility to the PBPK model developer, but more advanced modeling and programming skills and experience are required; thus, they are less suitable for beginners. However, both acslX and Berkeley Madonna software attempt to incorporate features to make them more intuitive for beginners by providing, for example, a graphical interface and a PBPK library to assemble models from already programmed code. PK-Sim: [http://www.systems-biology.com/products/pk-sim .html], Simcyp: [http://www.simcyp.com], and GastroPlus: [http://www.simulations-plus.com], which are specialized PBPK modeling software packages, provide less flexibility in 
model development, but they also require less mathematical and modeling experience. Such software tools provide the user with either a click-and-drag assembly of the model structure or an already built model, and can either simulate particular PK-relevant processes (e.g., intestinal absorption or metabolism) or constitute a generic whole body PBPK model. Many offer additional features; for example, Simcyp and PK-Sim allow simulation of complex absorption, distribution, metabolism, and excretion outcomes involving multiple drug interactions and parent drug and metabolite profiles. They also allow the simulation of virtual patient populations such as obese/morbidly obese individuals and patients with renal impairment or liver cirrhosis, and include a clearance prediction model that incorporates knowledge about growth, development, and maturation of various organs and tissues involved in drug metabolism and elimination across pediatric age groups to predict clearance in children using adult values [13, 24, 25]. However, although specialized PBPK modeling software packages do not require programming skills as does general purpose software, they are still relatively complex to use. This is not only due to the diversity of input options and many menus/windows that require knowledge about these parameters, where they are located, and how to work with them, but also because users of such software tools should have a substantial background knowledge of clinical pharmacology — for instance, pharmacokinetics topics as absorption, distribution, metabolism, and the elimination processes of drugs as well as pharmacogenetics and pharmacodynamics and also the molecular processes involved in these topics - to understand the difference among the various models offered and the model equations and assumptions so that the appropriate models are used to conduct PK analysis.

\subsection{PBPK Modeling Applications}

2.2.1. Overview. Although PBPK modeling was originally generated in the pharmaceutical field, it has more applications in environmental toxicology and risk assessment and has become a commonly and widely used technique. However, PBPK modeling can be used for a variety of other purposes and is becoming more popular in the fields of pharmacology and drug development $[6,11,12]$. Even during the early drug discovery programs and preclinical phase, PBPK modeling can be used, together with in vitro data and physicochemical characteristics, to anticipate the pharmacokinetics of potential drug candidates in animals, which may result in a reduction of unnecessary animal testing and significant time savings [26]. Another example demonstrated the utility of a PBPK/pharmacodynamic model in the selection of the most promising compound from five potential clinical candidates [27]. As PBPK models integrate relevant information from various sources, including those that are substance-dependent and physiologically relevant, they have been widely used for in IVIVE of drug kinetics through different species or routes of administration [27-29]. The PBPK-modeling technique has also emerged as a learning tool that can help users understand the influence of different processes and/or parameters involved in determining drug disposition and pharmacokinetic behavior [30]. PBPKmodeling is widely used in describing and/or predicting drug pharmacokinetic profiles by simulating different dosing regimens that allows established therapies to be evaluated and optimized. It also has been used to describe and/or predict drug pharmacokinetics under different physiological and pathological conditions; for example, in pregnancy [31, 32], under surgery [33], and in liver cirrhosis [34, 35]. The effects of food [36], aging [37, 38], rest and physical exertion [39-41], and gender differences [42] have also been explored using PBPK modeling. PBPK modeling can be used to estimate the pharmacokinetics of both a parent drug and its metabolite [43-45] and has been used successfully to predict the magnitude of complex drug-drug interactions and to clarify the change in drug pharmacokinetics upon concomitant drug administration [46-48], which is important within the pharmaceutical industry to improve safety and reduce the attrition rate of new drugs. Additionally, PBPK models are advantageous in that they enable prediction of exposure to a drug and/or toxics in the plasma or blood, but also in remote and/or inaccessible compartments such as the brain [49] or tumor tissues [50]. Quantified drug concentrations in different tissues and/or body fluids is easy to obtain as output when simulating and/or predicting using Simcyp or PK-Sim modeling software.

Three relevant aspects of PBPK modeling are presented in the following sections. Two examples are presented in more detail demonstrating how a PBPK model can be modified to describe drug pharmacokinetics under pathological or altered physiological conditions. Section 2.2.4. focuses on PBPK modeling in the pediatric population, with examples showing how an adult PBPK model is modified to an agespecific model to predict drug disposition in children and how pediatric clinical trials can benefit from such a modeling technique.

\subsubsection{Predicting and/or Describing of Drug Pharmacokinetics} in Individuals with Disease. Physiological changes associated with certain pathological conditions such as liver cirrhosis or renal insufficiency may affect the pharmacokinetic behavior of drugs. PBPK modeling emerges as a technique to predict drug pharmacokinetics under these conditions. One successful example is the study by Edginton and Willmann [34], in which they modified an existing "whole-body" PBPK model by incorporating physiological differences between healthy individuals and patients (changes in blood flow, reduction in plasma protein synthesis, reduced hepatic function, etc.) to predict drug pharmacokinetics in patients with liver cirrhosis. They searched the literature for quantitative measures of these physiological changes and included the following parameters: organ blood flows, cardiac index, plasma binding proteins, hematocrit, functional liver volume, hepatic enzymatic activity, and glomerular filtration rate. Table 1 summarizes the input values as reported in the paper. Finally, the modified model was used to predict the pharmacokinetic parameters and plasma concentration profiles for four compounds: alfentanil, lidocaine, theophylline, and levetiracetam. The results were then compared with literature data and found to be adequate. Figures 3 and 4 show the 
TABle 1: Physiological changes associated with liver cirrhosis (Child-Pugh score A-C) presented as fraction of control values from healthy individuals. The table is adapted from Edginton and Willmann [34].

\begin{tabular}{lccc}
\hline Parameter Child-Pugh class & & & \\
& $\mathrm{A}$ & $\mathrm{B}$ & $\mathrm{C}$ \\
\hline Blood flow & & & \\
$\quad$ Portal & 0.40 & 0.36 & 0.04 \\
$\quad$ Hepatic arterial & 1.3 & 2.3 & 3.4 \\
$\quad$ Renal & 0.88 & 0.65 & 0.48 \\
$\quad$ Other organs & 1.75 & 2.25 & 2.75 \\
Cardiac index & 1.11 & 1.27 & 1.36 \\
Albumin & 0.81 & 0.68 & 0.50 \\
$\alpha_{1}$-acid glycoprotein & 0.60 & 0.56 & 0.30 \\
Hematocrit value & 0.39 & 0.37 & 0.35 \\
Functional liver mass & 0.69 & 0.55 & 0.28 \\
Hepatic enzymes & & & \\
$\quad$ CYP3A4 & & 0.4 & 0.4 \\
$\quad$ CYP1A2 & 1 & 0.1 & 0.1 \\
$\quad$ CYP2E1 & 1 & 0.83 & 0.83 \\
GFR* & 1 & 0.70 & 0.36 \\
\hline
\end{tabular}

Fractions of control enzyme activity; CYP = cytochrome P450; ${ }^{*} \mathrm{GFR}=$ glomerular filtration rate.

simulation results for alfentanil and lidocaine, respectively. The modified model presented in this study indicates the ability of PBPK modeling to successfully account for altered physiology, and thus altered pharmacokinetics, in diseases when incorporating the eligible data. It can also serve as a building block for creating a generic/global whole-body PBPK model for the progressive disease of liver cirrhosis, which may facilitate future simulations and prediction of other drugs' pharmacokinetics.

\subsubsection{Predicting and/or Describing Drug Pharmacokinetics in} Individuals with Altered Physiology. Andrew et al.'s [32] study investigated the ability of PBPK modeling to assess drug pharmacokinetics in pregnant women. Midazolam was chosen as the initial study drug, because it has been well studied and prescribed during pregnancy. A coupled mother-fetus PBPK model for midazolam was developed in MATLAB with 20 maternal and 16 fetal tissue compartments connected via the placenta and fetal placental blood compartments. Model equations and input physiological and drug-specific parameters used in the model development were not reported in detail in this study; only their sources were mentioned. To validate the developed model, model prediction and observed data obtained from two studies for IV midazolam administered to six women following caesarian section were compared (Figure 5). Due to the lack of in vivo fetal data, model validation was limited to the adult side. However, despite limitations in the model validation, the developed model could serve as a starting point for building a coupled mother-fetus physiologically based model in humans to assess the effects of altered pharmacokinetics during pregnancy.

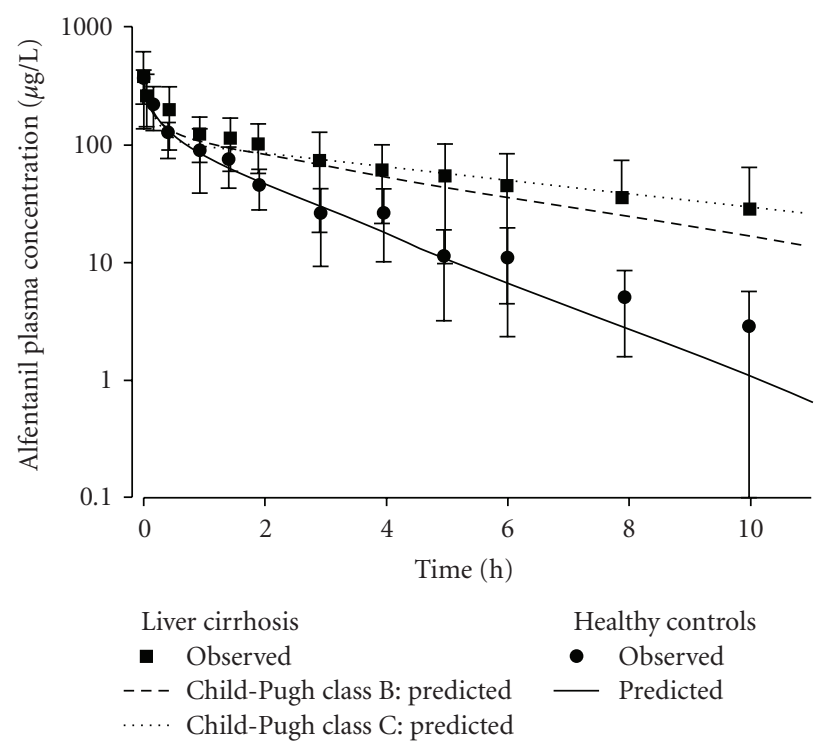

Figure 3: Predicted and observed arithmetic mean $( \pm \mathrm{SD})$ plasma concentration-time curves of alfentanil in (a) healthy controls and (b), (c) patients with liver cirrhosis. Figure is adapted from Edginton and Willmann [34].

2.2.4. PBPK Modeling in Pediatric Populations. Interest in in silico PBPK modeling in the field of pediatric dug development has been increasing since the initiation of the Pediatric Exclusivity Program in 1997 by the US FDA that was followed by the European Medicines Agency (EMA) in [51]. This is because PBPK modeling arises as a useful tool that can bridge pediatric and adult pharmacology. A childspecific PBPK model can deliver information about agedependent pharmacokinetic changes of drugs in children, explore "what if" scenarios to determine the most likely cause of altered pharmacokinetics, and potentially help in guiding pediatric clinical trials by suggesting, for example, first dose/dose range or optimal sampling times $[5,6]$.

Building a Pediatric PBPK Model to Perform Simulations and Predict Drug Pharmacokinetics in Children. A number of pediatric PBPK models have been developed to predict drug pharmacokinetics in children $[52,55,56]$, one of which is the model presented by Edginton et al. [52]. The aim of Edginton et al.'s study was to extend an existing adult PBPK model to reflect age-related physiological changes in children from birth to age eighteen. This age-modified model was then used together with a previously developed age-specific clearance model to predict pediatric plasma concentrations. To do this, the researchers used data from the literature, including agedependent changes of the relevant physiological parameters in children; for example, body weight, height, organ weights, blood flows, and interstitial and vascular space, to modify the adult model. Table 2 presents a sample of some physiological parameters used. They then selected five drugs with already available concentration-time profiles for both adults and children: paracetamol, theophylline, levofloxacin, alfentanil, and morphine. First, the pharmacokinetic profile of these 


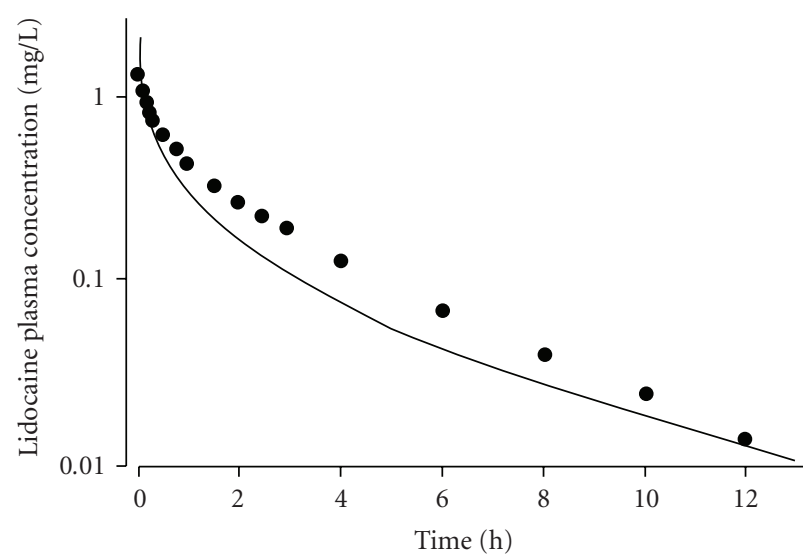

Healthy controls

- Observed Predicted

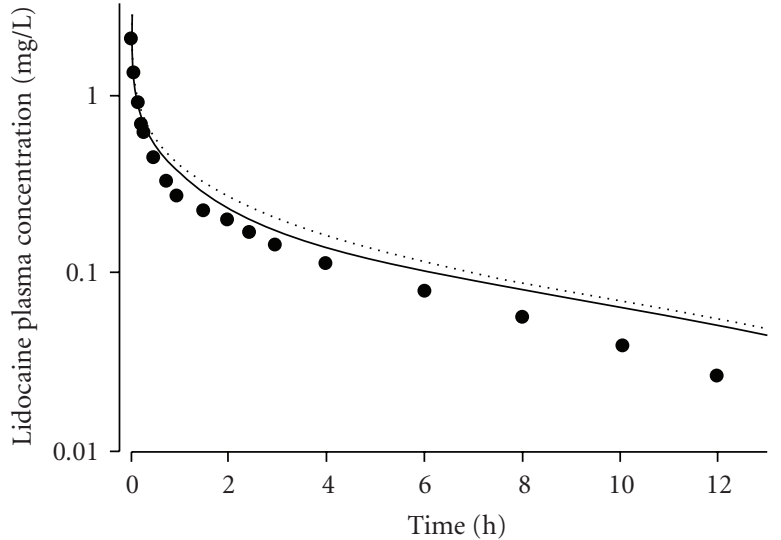

Child-Pugh class A

- Observed Predicted: using $f_{u}=0.42$ (calculated)

— Predicted: using $f_{u}=0.51$ (experimentally derived)

(b)

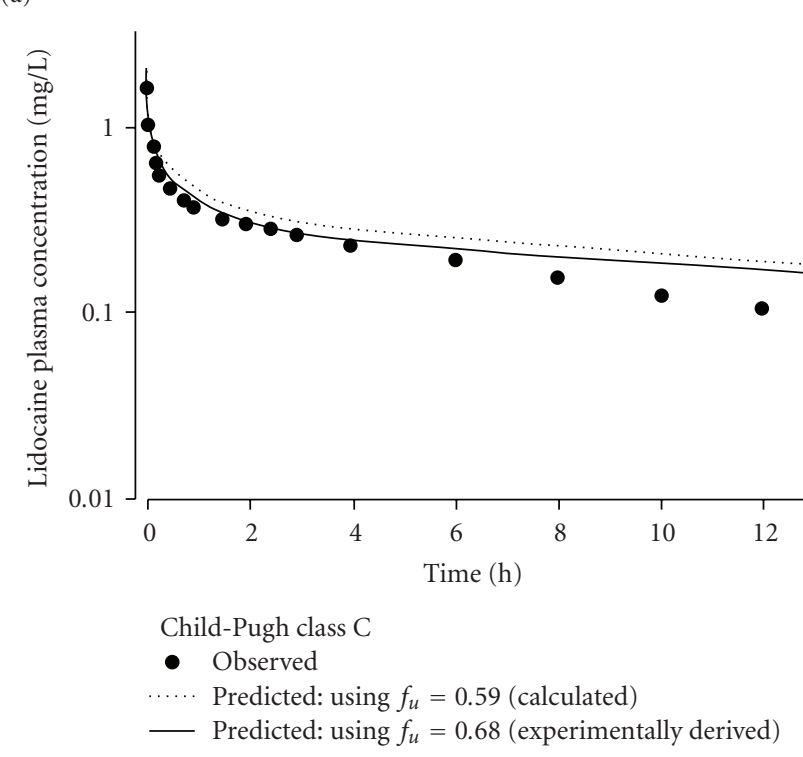

(c)

FIGURE 4: Comparison of lidocaine predicted and observed mean plasma concentration-time curves in healthy controls (a) and patients with Child-Pugh class A (b) and class C liver cirrhosis (c). Model simulation results adapted from Edginton and Willmann [34].

drugs was simulated in adults and compared with the data. When the simulated curves in adults matched the data with sufficient accuracy, a predicted pediatric clearance value for each drug was generated using the clearance model mentioned previously. Using this clearance value and the age-modified model, pediatric plasma concentrations were predicted. The predicted plasma concentration-time curves, volume of distribution $\left(V_{d}\right)$, and half-life $\left(t_{1 / 2}\right)$ were compared with the corresponding observed values to evaluate the accuracy of model predictions. Figure 6 provides an example of how well the predicted versus observed plasma concentrations for two drugs correlate. Another recent publication discussed a similar use of PBPK models in which Simcyp software was used to predict drug pharmacokinetics in children under various situations by performing simulations to replicate published pediatric clinical trials [7]. The same publication presented an interesting example, as a pediatric model was used to simulate a complex drug interaction scenario with a virtual 2-year-old child who was a patient in a pediatric intensive care unit. This pediatric model was used to investigate the effects of the interacting drugs in combination with midazolam pharmacokinetics. The simulations were able to show the effect on the plasma concentrations of midazolam (CYP 3A4 substrate) upon sequential adding of rifampicin (CYP 3A4 inducer), rifampicin and fluconazole (CYP 3A4 inhibitor), and rifampicin, fluconazole, and clarithromycin (CYP 3A4 inhibitor). This example illustrates the ability of PBPK modeling to address complex clinical drug-drug interactions and to explore "what if" scenarios. As a next step, these models need to be validated by prospective 


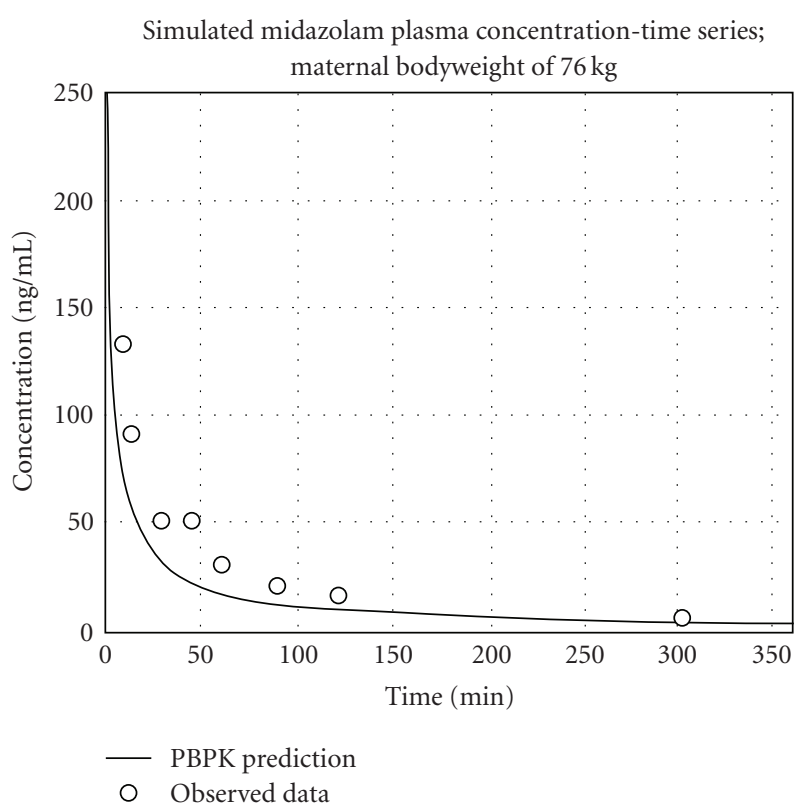

(a)

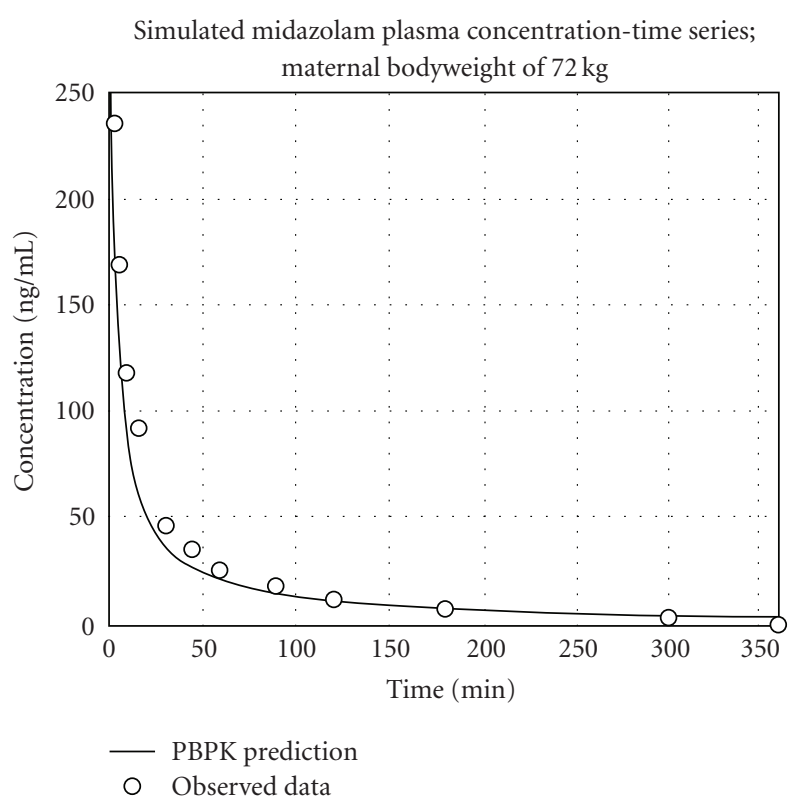

(b)

FIGURE 5: Simulated midazolam maternal plasma concentration-time profile following postcaesarean IV bolus compared with two different set of observed data; adapted from Andrew et al. [32].

TABLE 2: Values of organ blood flows, portal blood flow, total hepatic blood flow $(Q H)$, bodyweight, and height that were used in the PBPK model developed and reported by Edginton et al. [52].

\begin{tabular}{|c|c|c|c|c|c|c|}
\hline Parameter & New born & $1 \mathrm{y}$ & $5 y$ & $10 y$ & $15 y$ & Adult $(30 \mathrm{y})$ \\
\hline \multicolumn{7}{|l|}{ Organ blood flow (mL/min) } \\
\hline Adipose & 30 & 12 & 171 & 250 & $315 / 484^{\mathrm{a}}$ & $325 / 502^{\mathrm{a}}$ \\
\hline Brain & 180 & 700 & 900 & $840 / 750^{\mathrm{a}}$ & $805 / 708^{a}$ & $780 / 708^{\mathrm{a}}$ \\
\hline Gonads & 0.3 & 0.6 & $1.7 / 0.7^{\mathrm{a}}$ & $2.5 / 1.0^{\mathrm{a}}$ & $3.2 / 1.1^{\mathrm{a}}$ & $3.3 / 1.2^{\mathrm{a}}$ \\
\hline Heart & 24 & 48 & 136 & 200 & $252 / 285^{\mathrm{a}}$ & $260 / 295^{\mathrm{a}}$ \\
\hline Kidneys & 110 & 230 & 577 & 854 & $1335 / 950^{\mathrm{a}}$ & $1325 / 1120^{\mathrm{a}}$ \\
\hline Large intestine & 24 & 48 & 136 & 200 & $251 / 285^{\mathrm{a}}$ & $260 / 295^{\mathrm{a}}$ \\
\hline Liver & 39 & 78 & 221 & 325 & $409 / 370^{\mathrm{a}}$ & $423 / 383^{\mathrm{a}}$ \\
\hline Muscle & 31 & 72 & 212 & 429 & $941 / 646^{\mathrm{a}}$ & $1105 / 665^{\mathrm{a}}$ \\
\hline Pancreas & 6 & 12 & 34 & 50 & $63 / 57^{\mathrm{a}}$ & $65 / 59^{\mathrm{a}}$ \\
\hline Skeleton & 30 & 60 & 170 & 250 & $315 / 285^{\mathrm{a}}$ & $325 / 295^{\mathrm{a}}$ \\
\hline Skin & 30 & 60 & 170 & 250 & $315 / 285^{\mathrm{a}}$ & $325 / 295^{\mathrm{a}}$ \\
\hline Small intestine & 60 & 120 & 340 & 500 & $630 / 627^{\mathrm{a}}$ & $650 / 649^{\mathrm{a}}$ \\
\hline Spleen & 18 & 36 & 102 & 150 & $189 / 171^{\mathrm{a}}$ & $195 / 177^{\mathrm{a}}$ \\
\hline Stomach & 6 & 12 & 34 & 50 & $63 / 57^{\mathrm{a}}$ & $65 / 59^{\mathrm{a}}$ \\
\hline Portal blood flow (mL/min) & 114 & 228 & 646 & $950 / 950^{\mathrm{a}}$ & $1197 / 1197^{\mathrm{a}}$ & $1235 / 1239^{a}$ \\
\hline $\mathrm{QH}(\mathrm{mL} / \mathrm{min})^{*}$ & 153 & 306 & 867 & $1275 / 1273^{\mathrm{a}}$ & $1600 / 1566^{\mathrm{a}}$ & $1660 / 1620^{\mathrm{a}}$ \\
\hline Body weight (kg) & 3.5 & 10 & 19 & 32 & $56 / 53^{\mathrm{a}}$ & $73 / 60^{\mathrm{a}}$ \\
\hline Height $(\mathrm{cm})$ & 51 & 76 & 109 & 138 & $167 / 161^{\mathrm{a}}$ & $176 / 163^{\mathrm{a}}$ \\
\hline
\end{tabular}

$\mathrm{a}=$ Male/female; ${ }^{*} \mathrm{Q}_{H}=$ total hepatic blood flow.

pharmacokinetic data in children as an ultimate proof of their accuracy. Having qualified the models in such a way, "what if" scenarios could be simulated to support future trial designs or even guide individual drug treatments. In addition, developing and validating such pediatric PBPK models is important because of their potential to explore the pharmacokinetics of drugs for which little or no pediatric data are available.

Using the Pediatric PBPK Model to Suggest Dosing in Children. An example of how a pediatric PBPK model can be used to suggest age-specific doses for sildenafil was presented by 


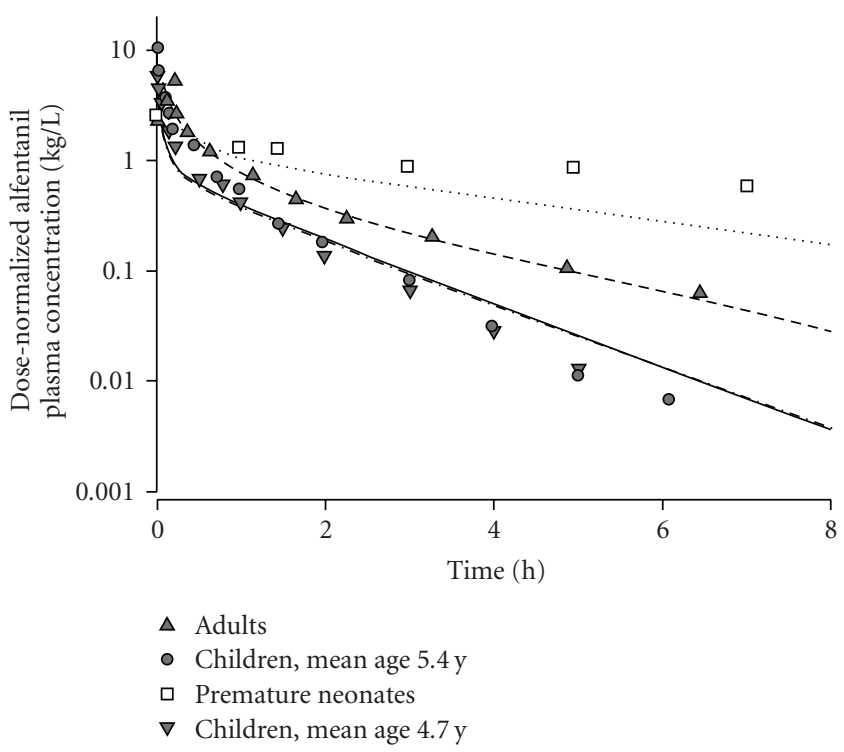

(a)

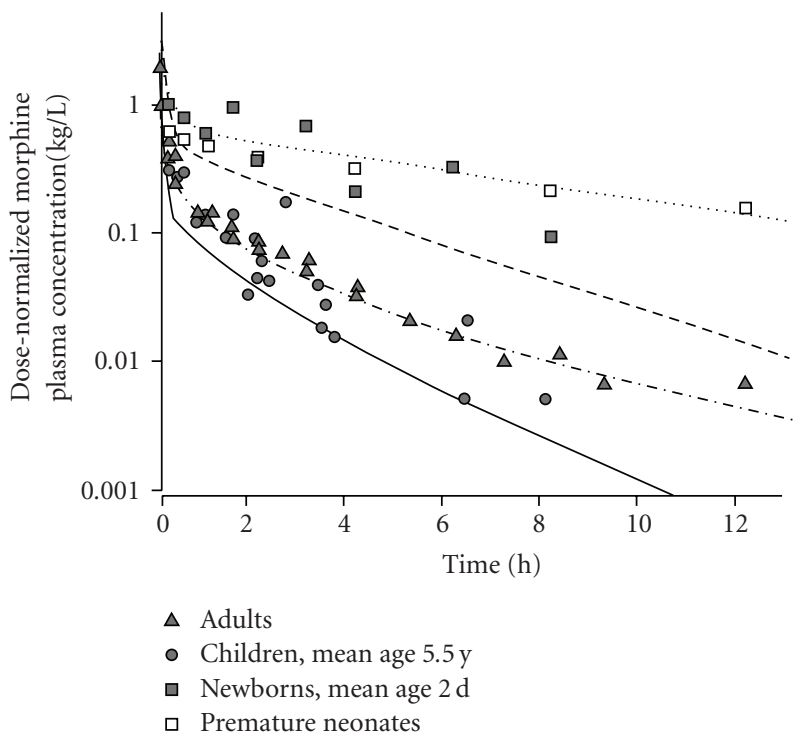

(b)

Figure 6: Predicted dose-normalized pediatric plasma concentration-time curves (lines) versus observed data (symbols) for (a) alfentanil and (b) morphine as reported in the original study by Edginton et al. [52]. Observed data was obtained from various studies in the literature.

Hsien [53]. Hsien developed and validated, in the same way as mentioned previously, a PBPK model for sildenafil in adults. A clearance scaling module was also used with an agemodified PBPK model to predict plasma concentrations of sildenafil in children that could supplement the very few pediatric pharmacokinetic data available in literature so far. The pediatric PBPK model of sildenafil was used to run simulations in virtual pediatric populations of different ages to estimate the exposure after a weight-normalized dose according to adult data. The simulation results showed that after 3 months of age, the exposure of the same weightnormalized dose across the pediatric age groups increased as the age of children increased and, therefore, that age-specific dose adaptation is necessary to achieve a relatively constant drug exposure in adults and children (Figure 7). The suggested dosing adjustments across pediatric age groups in this example of sildenafil have yet to be validated with prospectively collected data and are, therefore, hypothetical. Nevertheless, upon establishing and validating the use of PBPK modeling for this purpose, the provided data may help clinical trials become more "confirmatory" rather than "exploratory," and thus the modeling exercise will potentially save time, effort, and the number of trials needed to be performed in children.

Using the Pediatric PBPK Model to Suggest Sampling Times. Willmann [54] provides an example of using a PBPK model to determine optimal sampling times in children, comparing the possible difference between suggested pediatric sampling times based on adult concentration-time data and suggested sampling times based on simulations by a PBPK model (Figure 8). The latter, as shown in the study, can potentially help detect the best sampling times for a pharmacokinetic analysis and avoid taking blood samples where drug concentration is out of detection range. This is of great importance, especially in neonates and infants, as taking blood samples is more difficult than in adults and a main challenge in conducting pediatric clinical trials. The primary reason for using a PBPK model to provide such information in advance is to help conduct these trials optimally. Whether these techniques can reduce the number of children required for clinical trials and, therefore, save time, effort, and cost remains to be proven. Finally, it is worth mentioning that the ultimate gold standard is prospectively validating predictions/simulations generated by pediatric PBPK models. To our knowledge, in vivo data for validation up to now had been collected before the predictions of the models had been made. To qualify a model ultimately, data should be collected after predictions had been made and then compared. Unfortunately, there is a definite shortage of clinical examples that verify the use of PBPK models prospectively; such examples need to be developed to gain confidence in using PBPK models in clinical situations.

2.3. Limitations of $P B P K$ Modeling. PBPK modeling requires comprehensive data about the physiological, biochemical, and physicochemical processes that occur in biological systems in different age groups or under certain physiological and pathological conditions. These data are not available from only one source, which may lead to some confusion and to a problem in establishing a reliable source of accurate and consistent information. PBPK models reflect current scientific knowledge, and while some processes are known to be well characterized, others are partly or poorly characterized, as information gaps may exist. Information gaps or poor characterization of some physiological processes, 


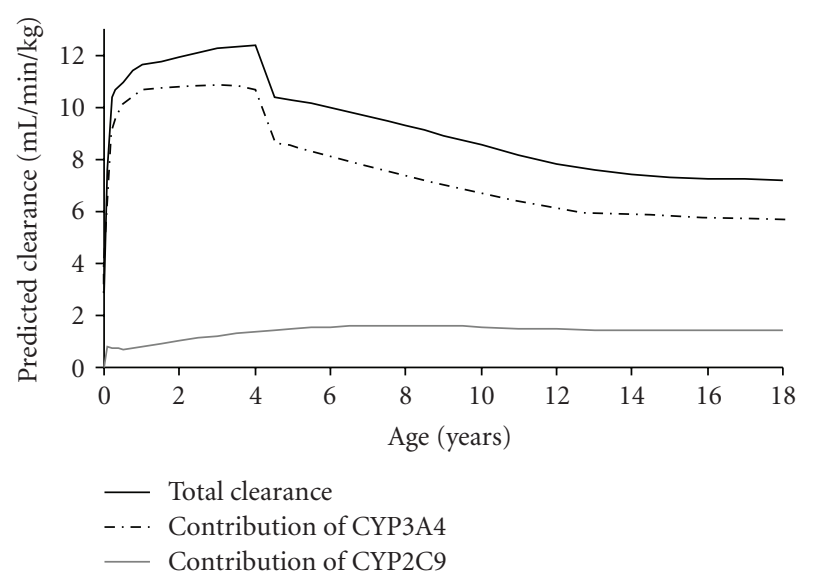

(a)
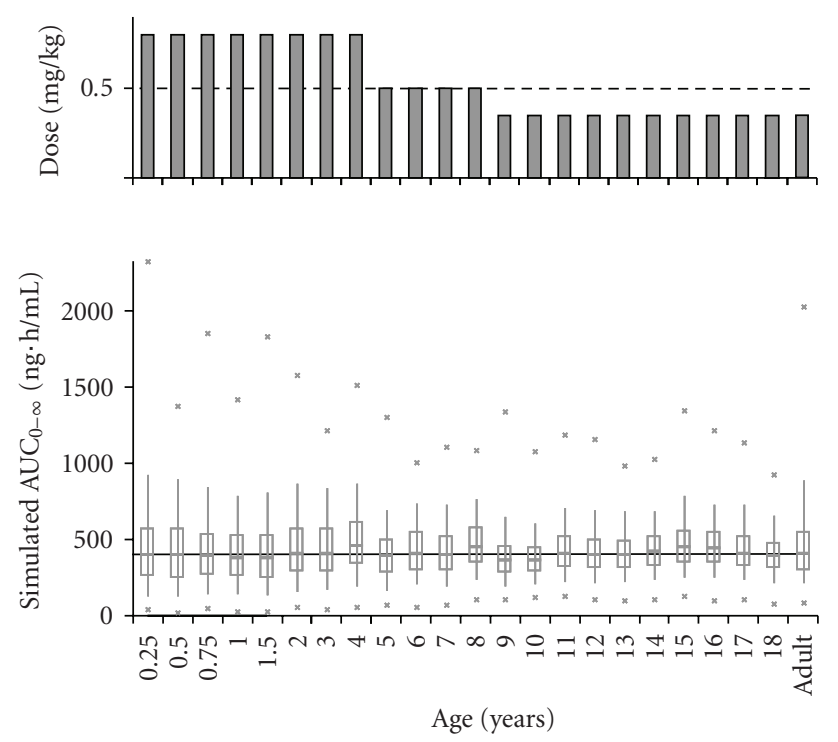

(b)

FIGURE 7: Simulation results using the PK-Sim software of a PBPK model for sildenafil in children [53]. (a): Predicted age-dependent sildenafil hepatic clearance across different pediatric ages based on the clearance scaling module in PK-Sim software. (b): Age-related doses for oral sildenafil in children depending on the simulated age-related exposure (not shown) of sildenafil in a virtual pediatric population and the estimated exposure of the estimated doses of sildenafil in children between 3 months and 18 years. Potential pediatric doses based on simulations for sildenafil to achieve adult exposure: infants and children from 3 months to 4 years: $0.8 \mathrm{mg} / \mathrm{kg}$; children from 5 to 8 years: $0.5 \mathrm{mg} / \mathrm{kg}$; and children older than 8 years: $0.35 \mathrm{mg} / \mathrm{kg}$ as in adults. Box plots represent median, 25th, and 75th percentiles (box), 5th and 95th percentiles (error bar), and maximum and minimum values $(x)$ of $\mathrm{AUC}_{0-\infty}$ from 1000 simulations in each age group.

such as transporters' abundance or the absorption process in newborns and infants, may cause the model to fail to optimally describe the pharmacokinetic behavior of some drugs in such population. Therefore, it is important to emphasize that the validity and quality of the simulations depends on the corresponding model and its incorporated data as well as its purpose, and uncertainty concerning the

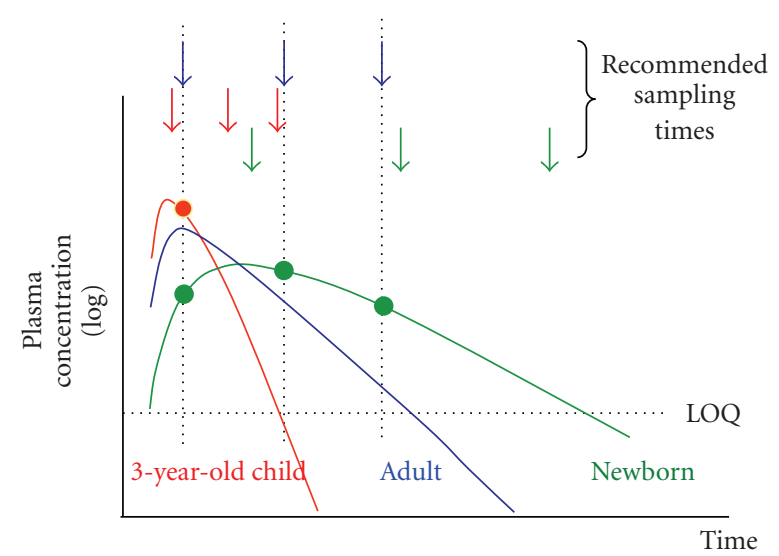

FIGURE 8: Schematic drawing of a potential application of PBPK simulations for children of different ages to find optimal blood sampling time points for the pharmacokinetic investigations in a future pediatric trial according to Willmann [54]. Arrows indicate optimal sampling time for a 3-year-old child, a newborn, and an adult. LOQ: limit of quantification.

data used to build the model should be properly reflected. Moreover, as simulations are associated with prediction errors and uncertainty, they require accurate judgment and interpretation of their inferences to frame them in the right context. In addition, simulation results should be supported by experimental data, and should not be used to replace data from well conducted studies as primary degree of evidence [57]. Care should be given to the fact that poor quality modeling and simulations practices could lead to a biased model or overestimation of the predictive power of the model. An extensive and continuous evaluation of the model will help minimize and identify early the biased models. Finally, the researcher/user of this modeling technique should understand the physiological and pharmacological rationale behind the model and should be aware that PBPK modeling, despite its potential benefits and various implementations, does not provide the ultimate solution and that there remains a shortage of prospective examples that verify that this technique is as good in clinical practice as in theory.

\section{Conclusion}

This paper provides a brief overview that covers the methodology, applications and limitations of PBPK modeling with special attention given to discuss use of PBPK models in pediatric drug development with some examples described in detail. The applications of the PBPK modeling technique are diverse, as PBPK models can potentially be used at different stages of drug development from early discovery phases and preclinical development up to clinical phase studies. Simulations by PBPK models are developed to potentially save time and effort, to explore the effect of various factors on drug pharmacokinetics, to address the magnitude of drug-drug interactions, and to help optimize conductance of clinical trials in special populations such as pediatrics, where optimal planning is needed to minimize the ethical 
and technical difficulties and is, therefore, an essential key for success. However, prospective examples that assure the clinical value of such a modeling technique are needed and are important to increase the acceptance of these techniques in the planning phases of clinical trials or for the practice of the individual drug treatment. PBPK models do have limitations and weaknesses. For instance, because they reflect current scientific knowledge, some physiological processes are poorly characterized and information gaps may exist. In addition, the validity of the simulations depends on the corresponding model and its incorporated data, and obtained results are associated with prediction errors and uncertainty. The use of PBPK modeling technique is still relatively narrow, but the introduction of many universally applicable software tools with a more user-friendly interface that do not require an extensive modeling and/or mathematical background have facilitated its use and contributed significantly to its wider implementation in different scientific areas over the last few years. Researchers need, however, to have a substantial background knowledge of clinical pharmacology, pharmacokinetics, and pharmacodynamics as well as the molecular processes involved in these topics to understand the difference among the various models offered and the model equations and assumptions so that the appropriate models are used to conduct PK analysis and to accurately interpret and judge the results.

\section{References}

[1] I. Nestorov, "Whole-body physiologically based pharmacokinetic models," Expert Opinion on Drug Metabolism and Toxicology, vol. 3, no. 2, pp. 235-249, 2007.

[2] H.-S. G. Chen and J. F. Gross, "Physiologically based pharmacokinetic models for anticancer drugs," Cancer Chemotherapy and Pharmacology, vol. 2, no. 2, pp. 85-94, 1979.

[3] K. J. Himmelstein and R. J. Lutz, "A review of the applications of physiologically based pharmacokinetic modeling," Journal of Pharmacokinetics and Biopharmaceutics, vol. 7, no. 2, pp. 127-145, 1979.

[4] I. Nestorov, "Whole body pharmacokinetic models," Clinical Pharmacokinetics, vol. 42, no. 10, pp. 883-908, 2003.

[5] G. M. Grass and P. J. Sinko, "Physiologically-based pharmacokinetic simulation modelling," Advanced Drug Delivery Reviews, vol. 54, no. 3, pp. 433-451, 2002.

[6] N. Parrott and T. Lave, "Applications of physiologically based absorption models in drug discovery and development," Molecular Pharmaceutics, vol. 5, no. 5, pp. 760-775, 2008.

[7] T. N. Johnson and A. Rostami-Hodjegan, "Resurgence in the use of physiologically based pharmacokinetic models in pediatric clinical pharmacology: parallel shift in incorporating the knowledge of biological elements and increased applicability to drug development and clinical practice," Paediatric Anaesthesia, vol. 21, no. 3, pp. 291-301, 2011.

[8] F. Bouzom and B. Walther, "Pharmacokinetic predictions in children by using the physiologically based pharmacokinetic modelling," Fundamental and Clinical Pharmacology, vol. 22, no. 6, pp. 579-587, 2008.

[9] F. Y. Bois, "Physiologically based modelling and prediction of drug interactions," Basic and Clinical Pharmacology and Toxicology, vol. 106, no. 3, pp. 154-161, 2010.
[10] C. Lupfert and A. Reichel, "Development and application of physiologically based pharmacokinetic-modeling tools to support drug discovery," Chemistry and Biodiversity, vol. 2, no. 11, pp. 1462-1486, 2005.

[11] M. Rowland, C. Peck, and G. Tucker, "Physiologically-based pharmacokinetics in drug development and regulatory science," Annual Review of Pharmacology and Toxicology, vol. 51, pp. 45-73, 2011.

[12] P. Espié, D. Tytgat, M. L. Sargentini-Maier, I. Poggesi, and J. B. Watelet, "Physiologically based pharmacokinetics (PBPK)," Drug Metabolism Reviews, vol. 41, no. 3, pp. 391-407, 2009.

[13] S. Willmann, J. Lippert, M. Sevestre, J. Solodenko, F. Fois, and W. Schmitt, "PK-Sim®: a physiologically based pharmacokinetic 'whole-body' model," Biosilico, vol. 1, no. 4, pp. 121-124, 2003.

[14] K. B. Bischoff, "Some fundamental considerations of the applications of pharmacokinetics to cancer chemotherapy," Cancer Chemotherapy Reports, vol. 59, no. 4, pp. 777-793, 1975.

[15] I. A. Nestorov, L. J. Aarons, P. A. Arundel, and M. Rowland, "Lumping of whole-body physiologically based pharmacokinetic models," Journal of Pharmacokinetics and Biopharmaceutics, vol. 26, no. 1, pp. 21-46, 1998.

[16] C. Brochot, J. Toth, and F. Y. Bois, "Lumping in pharmacokinetics," Journal of Pharmacokinetics and Pharmacodynamics, vol. 32, no. 5-6, pp. 719-736, 2005.

[17] J. Valentin, "Basic anatomical and physiological data for use in radiological protection: reference values: ICRP Publication 89," Annals of the ICRP, vol. 32, no. 3-4, pp. 1-277, 2002.

[18] R. P. Brown, M. D. Delp, S. L. Lindstedt, L. R. Rhomberg, and R. P. Beliles, "Physiological parameter values for physiologically based pharmacokinetic models," Toxicology and Industrial Health, vol. 13, no. 4, pp. 407-484, 1997.

[19] Y. Sasaki and H. N. Wagner Jr., "Measurement of the distribution of cardiac output in unanesthetized rats," Journal of Applied Physiology, vol. 30, no. 6, pp. 879-884, 1971.

[20] D. R. Wada, H. Harashima, W. F. Ebling, E. W. Osaki, and D. R. Stanski, "Effects of thiopental on regional blood flows in the rat," Anesthesiology, vol. 84, no. 3, pp. 596-604, 1996.

[21] P. Poulin and F.-P. Theil, "A priori prediction of tissue: plasma partition coefficients of drugs to facilitate the use of physiologically-based pharmacokinetic models in drug discovery," Journal of Pharmaceutical Sciences, vol. 89, no. 1, pp. 16-35, 2000.

[22] T. Rodgers and M. Rowland, "Physiologically based pharmacokinetic modelling 2: predicting the tissue distribution of acids, very weak bases, neutrals and zwitterions," Journal of Pharmaceutical Sciences, vol. 95, no. 6, pp. 1238-1257, 2006.

[23] W. Schmitt, "General approach for the calculation of tissue to plasma partition coefficients," Toxicology In Vitro, vol. 22, no. 2, pp. 457-467, 2008.

[24] M. Jamei, S. Marciniak, K. Feng, A. Barnett, G. Tucker, and A. Rostami-Hodjegan, "The simcyp population-based ADME simulator," Expert Opinion on Drug Metabolism and Toxicology, vol. 5, no. 2, pp. 211-223, 2009.

[25] S. Willmann, J. Lippert, and W. Schmitt, "From physicochemistry to absorption and distribution: predictive mechanistic modelling and computational tools," Expert Opinion on Drug Metabolism \& Toxicology, vol. 1, no. 1, pp. 159-168, 2005.

[26] M. Germani, P. Crivori, M. Rocchetti et al., "Evaluation of a basic physiologically based pharmacokinetic model for simulating the first-time-in-animal study," European Journal of Pharmaceutical Sciences, vol. 31, no. 3-4, pp. 190-201, 2007. 
[27] N. Parrott, H. Jones, N. Paquereau, and T. Lavé, "Application of full physiological models for pharmaceutical drug candidate selection and extrapolation of pharmacokinetics to man," Basic and Clinical Pharmacology and Toxicology, vol. 96, no. 3, pp. 193-199, 2005.

[28] S. S. De Buck and C. E. Mackie, "Physiologically based approaches towards the prediction of pharmacokinetics: in vitro-in vivo extrapolation," Expert Opinion on Drug Metabolism and Toxicology, vol. 3, no. 6, pp. 865-878, 2007.

[29] M. R. Shiran, N. J. Proctor, E. M. Howgate, K. Rowland-Yeo, G. T. Tucker, and A. Rostami-Hodjegan, "Prediction of metabolic drug clearance in humans: in vitro-in vivo extrapolation vs allometric scaling," Xenobiotica, vol. 36, no. 7, pp. 567-580, 2006.

[30] H. Sun and K. S. Pang, "Physiological modeling to understand the impact of enzymes and transporters on drug and metabolite data and bioavailability estimates," Pharmaceutical Research, vol. 27, no. 7, pp. 1237-1254, 2010.

[31] S. M. Hays, B. A. Elswick, G. M. Blumenthal, F. Welsch, R. B. Conolly, and M. L. Gargas, "Development of a physiologically based pharmacokinetic model of 2- methoxyethanol and 2methoxyacetic acid disposition in pregnant rats," Toxicology and Applied Pharmacology, vol. 163, no. 1, pp. 67-74, 2000.

[32] M. A. Andrew, M. F. Hebert, and P. Vicini, "Physiologically based pharmacokinetic model of midazolam disposition during pregnancy," in Proceedings of the 30th Annual International Conference of the IEEE Engineering in Medicine and Biology Society, pp. 5454-5457, 2008.

[33] S. Bjorkman, D. R. Wada, B.-M. Berling, and G. Benoni, "Prediction of the disposition of midazolam in surgical patients by a physiologically based pharmacokinetic model," Journal of Pharmaceutical Sciences, vol. 90, no. 9, pp. 12261241, 2001.

[34] A. N. Edginton and S. Willmann, "Physiology-based simulations of a pathological condition: prediction of pharmacokinetics in patients with liver cirrhosis," Clinical Pharmacokinetics, vol. 47, no. 11, pp. 743-752, 2008.

[35] T. N. Johnson, K. Boussery, K. Rowland-Yeo, G. T. Tucker, and A. Rostami-Hodjegan, "A semi-mechanistic model to predict the effects of liver cirrhosis on drug clearance," Clinical Pharmacokinetics, vol. 49, no. 3, pp. 189-206, 2010.

[36] H. M. Jones, N. Parrott, G. Ohlenbusch, and T. Lave, "Predicting pharmacokinetic food effects using biorelevant solubility media and physiologically based modelling," Clinical Pharmacokinetics, vol. 45, no. 12, pp. 1213-1226, 2006.

[37] J. Li and P. R. Gwilt, "The effect of age on the early disposition of doxorubicin," Cancer Chemotherapy and Pharmacology, vol. 51, no. 5, pp. 395-402, 2003.

[38] F. Yang, X. Tong, D. G. McCarver, R. N. Hines, and D. A. Beard, "Population-based analysis of methadone distribution and metabolism using an age-dependent physiologically based pharmacokinetic model," Journal of Pharmacokinetics and Pharmacodynamics, vol. 33, no. 4, pp. 485-518, 2006.

[39] J. E. Dennison, P. L. Bigelow, M. M. Mumtaz, M. E. Andersen, I. D. Dobrev, and R. S. H. Yang, "Evaluation of potential toxicity from co-exposure to three CNS depressants (toluene, ethylbenzene, and xylene) under resting and working conditions using PBPK modeling," Journal of Occupational and Environmental Hygiene, vol. 2, no. 3, pp. 127-135, 2005.

[40] G. Hamelin, G. Charest-Tardif, G. Truchon, and R. Tardif, "Physiologically based modeling of $\mathrm{n}$-hexane kinetics in humans following inhalation exposure at rest and under physical exertion: impact on free 2,5-hexanedione in urine and on n-hexane in alveolar air," Journal of Occupational and Environmental Hygiene, vol. 2, no. 2, pp. 86-97, 2005.

[41] M. B. Reddy, M. E. Andersen, P. E. Morrow et al., "Physiological modeling of inhalation kinetics of octamethylcyclotetrasiloxane in humans during rest and exercise," Toxicological Sciences, vol. 72, no. 1, pp. 3-18, 2003.

[42] H. J. Clewell, P. R. Gentry, T. R. Covington, R. Sarangapani, and J. G. Teeguarden, "Evaluation of the potential impact of age- and gender-specific pharmacokinetic differences on tissue dosimetry," Toxicological Sciences, vol. 79, no. 2, pp. 381-393, 2004.

[43] H. J. Clewell III, P. R. Gentry, T. R. Covington, and J. M. Gearhart, "Development of a physiologically based pharmacokinetic model of trichloroethylene and its metabolites for use in risk assessment," Environmental Health Perspectives, vol. 108, supplement 2, pp. 283-305, 2000.

[44] H. J. Clewell III, P. R. Gentry, J. M. Gearhart, T. R. Covington, M. I. Banton, and M. E. Andersen, "Development of a physiologically based pharmacokinetic model of isopropanol and its metabolite acetone," Toxicological Sciences, vol. 63, no. 2, pp. 160-172, 2001.

[45] H. A. El-Masri and E. M. Kenyon, "Development of a human physiologically based pharmacokinetic (PBPK) model for inorganic arsenic and its mono- and di-methylated metabolites," Journal of Pharmacokinetics and Pharmacodynamics, vol. 35, no. 1, pp. 31-68, 2008.

[46] K. Rowland Yeo, M. Jamei, J. Yang, G. T. Tucker, and A. Rostami-Hodjegan, "Physiologically based mechanistic modelling to predict complex drug-drug interactions involving simultaneous competitive and time-dependent enzyme inhibition by parent compound and its metabolite in both liver and gut-the effect of diltiazem on the time-course of exposure to triazolam," European Journal of Pharmaceutical Sciences, vol. 39, no. 5, pp. 298-309, 2010.

[47] M. Vossen, M. Sevestre, C. Niederalt, I. J. Jang, S. Willmann, and A. N. Edginton, "Dynamically simulating the interaction of midazolam and the CYP3A4 inhibitor itraconazole using individual coupled whole-body physiologically-based pharmacokinetic (WB-PBPK) models," Theoretical Biology and Medical Modelling, vol. 4, article 13, 2007.

[48] X. Zhang, S. K. Quinney, J. C. Gorski, D. R. Jones, and S. D. Hall, "Semiphysiologically based pharmacokinetic models for the inhibition of midazolam clearance by diltiazem and its major metabolite," Drug Metabolism and Disposition, vol. 37, no. 8, pp. 1587-1597, 2009.

[49] X. Liu, B. J. Smith, C. Chen et al., "Use of a physiologically based pharmacokinetic model to study the time to reach brain equilibrium: an experimental analysis of the role of bloodbrain barrier permeability, plasma protein binding, and brain tissue binding," Journal of Pharmacology and Experimental Therapeutics, vol. 313, no. 3, pp. 1254-1262, 2005.

[50] J. M. Gallo, P. Vicini, A. Orlansky et al., "Pharmacokinetic model-predicted anticancer drug concentrations in human tumors," Clinical Cancer Research, vol. 10, no. 23, pp. 80488058, 2004.

[51] European Union, "Regulation (EC) no 1901/2006 of the European Parliament and of the council of 12 december 2006 on medicinal products for paediatric use and amending regulation (EEC) no 1768/92, directive 2001/20/EC, directive 2001/83/EC and regulation (EC) no 726/2004".

[52] A. N. Edginton, W. Schmitt, and S. Willmann, "Development and evaluation of a generic physiologically based pharmacokinetic model for children," Clinical Pharmacokinetics, vol. 45, no. 10, pp. 1013-1034, 2006. 
[53] L. Hsien, Identifying paediatric needs in cardiology and the prediction of sildenafil exposure in children with pulmonary arterial hypertension, Inauguraldissertation, Faculty of Mathematics and Sciences, University of Düsseldorf, Düsseldorf, Germany, 2010.

[54] S. Willmann, "Can computer simulations replace clinical pharmacokinetic," Pharmazie in Unserer Zeit, vol. 38, no. 1, pp. $62-67,2009$.

[55] G. Ginsberg, D. Hattis, A. Russ, and B. Sonawane, "Physiologically based pharmacokinetic (PBPK) modeling of caffeine and theophylline in neonates and adults: implications for assessing children's risks from environmental agents," Journal of Toxicology and Environmental Health, vol. 67, no. 4, pp. 297329, 2004.

[56] S. Bjorkman, "Prediction of drug disposition in infants and children by means of physiologically based pharmacokinetic (PBPK) modelling: theophylline and midazolam as model drugs," British Journal of Clinical Pharmacology, vol. 59, no. 6, pp. 691-704, 2005.

[57] E. Manolis and G. Pons, "Proposals for model-based paediatric medicinal development within the current European Union regulatory framework," British Journal of Clinical Pharmacology, vol. 68, no. 4, pp. 493-501, 2009. 

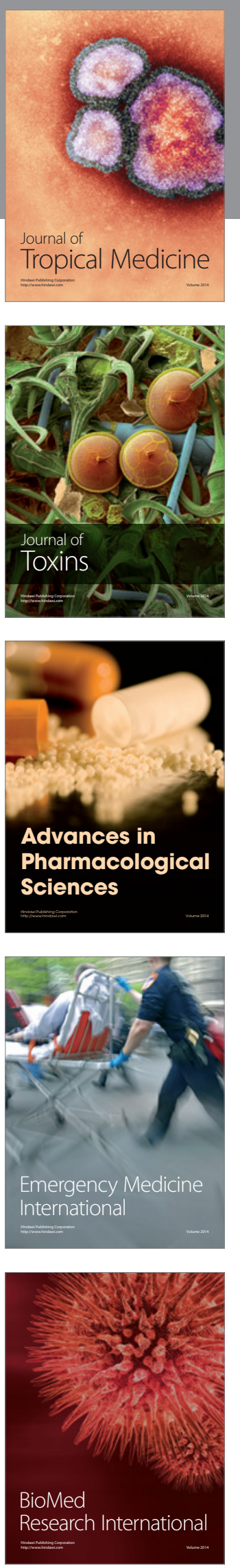
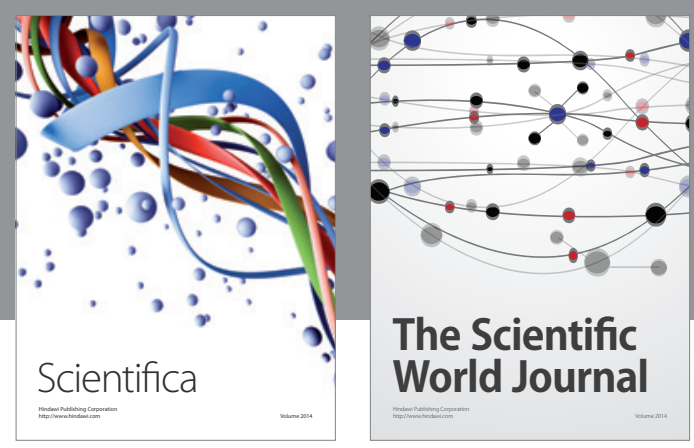

The Scientific World Journal
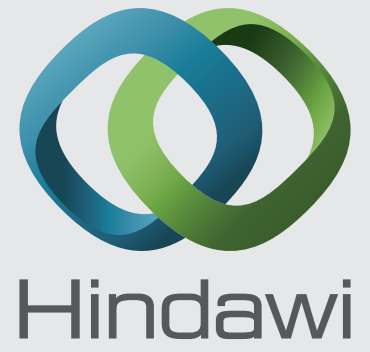

Submit your manuscripts at

http://www.hindawi.com
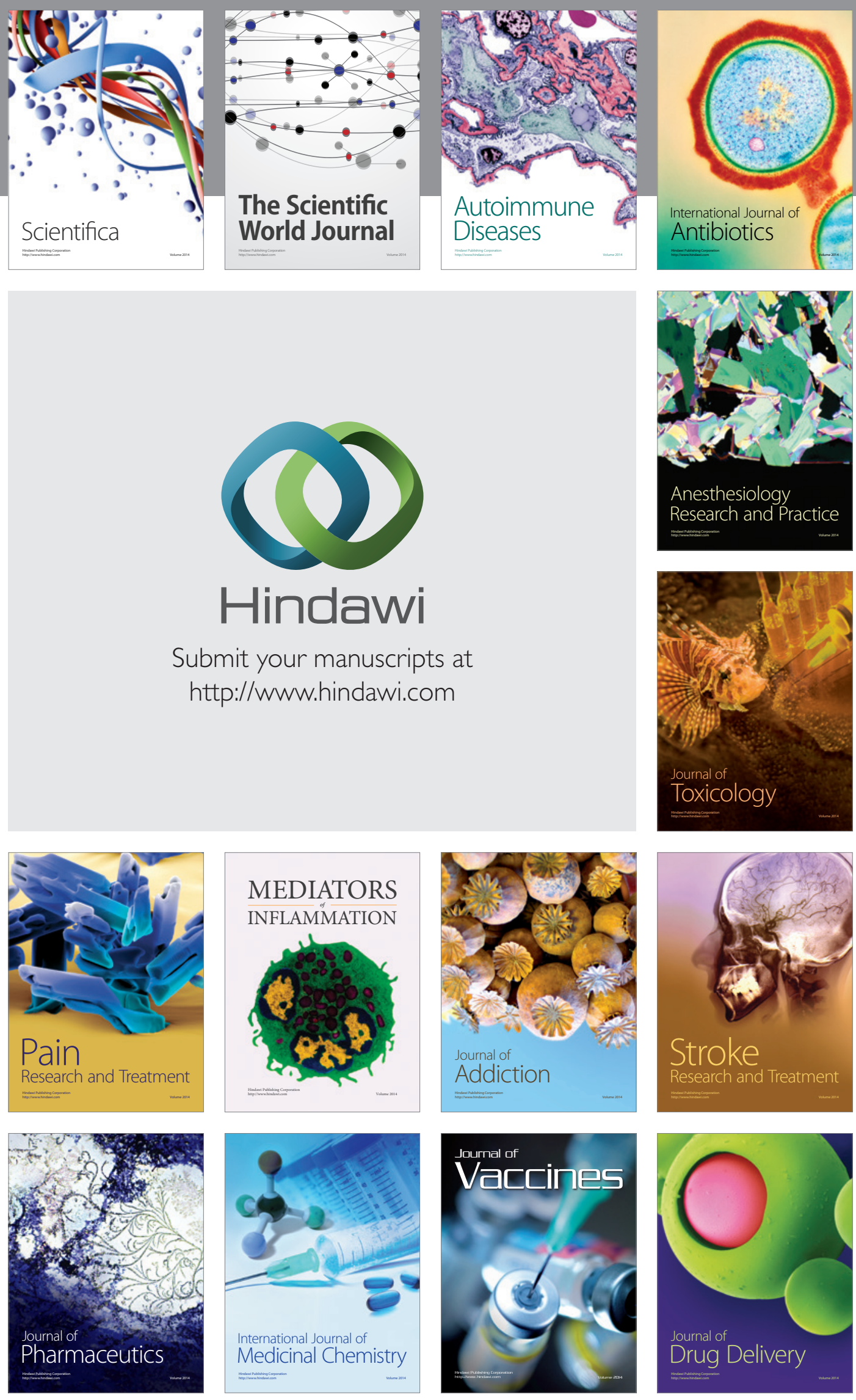